\title{
Electromagnetic Response of the BCS Superconductor: Flow Equation Approach
}

\author{
M. ZAPAlSKa AND T. DOMAŃSKi \\ Institute of Physics, M. Curie Sklodowska University, Radziszewskiego 10, 20-031 Lublin, Poland \\ We address the linear response for superconductors using the non-perturbative technique based on the \\ continuous unitary transformation. We discuss how this method rigorously reproduces the standard BCS solution \\ and point out how such result can be generalized to the case of electron systems with strong pairing fluctuations \\ and in presence of the nonequilibrium phenomena.
}

PACS: 74.20.Fg, 71.10.Li, 05.10.Cc

\section{Introduction}

The flow equation approach introduced in a context of the condensed matter by F. Wegner [1] and in a context of the high energy physics by K. Wilson with S. Głazek [2] proved to be very useful tool for studying a number of problems in physics [3]. Its main idea is based on a continuous unitary transformation which in a sequence of infinitesimal steps converts the initial Hamiltonian to a diagonal or block-diagonal structure. The unitary flow of the Hamiltonian is generated by an antihermitean operator $\hat{\eta}$ which can be choosen in a somewhat arbitrary way. The most convenient choice of such generator is usually dictated by the specific problem under consideration [3].

In this paper we discuss the continuous unitary transformation used for digonalization of the BCS model which can be further extended to various non-trivial models, where the strong electron correlations play an important role. We focus on the technical details concerning determination of the current-current response function for the BCS superconducting which have been omitted in our previous work [4]. We also point out how this treatment can be used for studying the fluctuations and various non-equilibrium phenomena.

\section{The flow equation approach}

Let us briefly sketch the main idea how the flow equation method deals with an arbitrary Hamiltonian $\hat{H}$

$$
\hat{H}=\hat{H}_{0}+\hat{H}_{\text {int }},
$$

consisting of the diagonal $\hat{H}_{0}$ and the interactions terms $\hat{H}_{\text {int }}$ (the latter part can describe any kind of interactions or perturbations). Diagonalization of the Hamiltonian is achieved by the continuous unitary transformation $\hat{H}(l)=\hat{U}^{\dagger}(l) \hat{H} \hat{U}(l)$ (where $l$ is a formal flow parameter). Evolution of the Hamiltonian is governed by the flow equation [1]

$$
\frac{\mathrm{d} \hat{H}(l)}{\mathrm{d} l}=[\hat{\eta}(l), \hat{H}(l)],
$$

where the canonical generator is defined as $\hat{\eta}(l) \equiv$ $\frac{\mathrm{d} \hat{U}(l)}{\mathrm{d} l} \hat{U}^{-1}(l)$. For the case without any degenerate states in the system Wegner proposed the following convenient generating operator [1]

$$
\hat{\eta}(l)=\left[\hat{H}_{0}(l), \hat{H}_{\text {int }}(l)\right] .
$$

This choice (3) guarantees the asymptotic flow to a diagonal structure $\lim _{l \rightarrow \infty} \hat{H}_{\text {int }}(l)=0$.

In the many particle systems we usually need to determine various statistical averages of the observables

$$
\langle\hat{O}\rangle_{\hat{H}}=\operatorname{Tr}\left(\mathrm{e}^{-\beta \hat{\mathrm{H}}} \hat{\mathrm{O}}\right) / \operatorname{Tr}\left(\mathrm{e}^{-\beta \hat{\mathrm{H}}}\right),
$$

where $\beta^{-1}=k_{B} T$. Using invariance of the trace on the unitary transformations $\hat{O}(l)=\hat{U}(l) \hat{O} \hat{U}^{-1}(l)$ one has then to analyze the flow of individual observables $\hat{O} \rightarrow \hat{O}(l) \rightarrow \hat{O}(\infty)$ according to the differential equation [1]

$$
\frac{\mathrm{d} \hat{O}(l)}{\mathrm{d} l}=[\hat{\eta}(l), \hat{O}(l)]
$$

besides the flow (2) of the Hamiltonian $\hat{H}(l)$.

\section{BCS problem}

To illustrate how the flow equation procedure reproduces the BCS solution let us consider the bilinear Hamiltonian describing electrons coupled to the classical pairing field $\Delta_{k}$

$$
\hat{H}=\sum_{\boldsymbol{k}, \sigma} \xi_{\boldsymbol{k}} \hat{c}_{\boldsymbol{k} \sigma}^{\dagger} \hat{c}_{\boldsymbol{k} \sigma}-\sum_{\boldsymbol{k}}\left(\Delta_{\boldsymbol{k}} \hat{c}_{\boldsymbol{k} \uparrow}^{\dagger} \hat{c}_{-\boldsymbol{k} \downarrow}^{\dagger}+\text { h.c. }\right),
$$

where $\hat{c}_{\boldsymbol{k} \sigma}^{\dagger}\left(\hat{c}_{\boldsymbol{k} \sigma}\right)$ is the creation (annihilation) operator, $\xi_{\boldsymbol{k}}=\varepsilon_{\boldsymbol{k}}-\mu$ is the energy measured with respect to the chemical potential $\mu$. We can regard the classical field $\Delta_{k}$ as Bose-Einstein condensate of the Cooper pairs $\Delta_{\boldsymbol{k}}=-\sum_{\boldsymbol{q}} V_{\boldsymbol{k}, \boldsymbol{q}}\left\langle\hat{c}_{-\boldsymbol{q} \downarrow} \hat{c}_{\boldsymbol{q} \uparrow}\right\rangle$ formed by an attractive potential $V_{\boldsymbol{k}, \boldsymbol{q}}<0$. The present procedure can be generalized on the nontrivial case when $\Delta_{k}$ is treated as the quantum field [4].

According to (3), we obtain for the BCS model (6) the following canonical generator

$$
\begin{aligned}
& \hat{\eta}(l)=2 \sum_{\boldsymbol{k}} \xi_{\boldsymbol{k}}(l)\left(\Delta_{\boldsymbol{k}}(l) \hat{c}_{\boldsymbol{k} \uparrow}^{\dagger} \hat{c}_{-\boldsymbol{k} \downarrow}^{\dagger}\right. \\
& \left.-\Delta_{\boldsymbol{k}}^{*}(l) \hat{c}_{-\boldsymbol{k} \downarrow} \hat{c}_{\boldsymbol{k} \uparrow}\right) .
\end{aligned}
$$

Transformation of the Hamiltonian $\hat{H}(l)$ proceeds as long 
as $\eta(l)$ is finite, which occurs until $\Delta_{\boldsymbol{k}}(l) \rightarrow 0$. This is achieved in the asymptotic limit $l \rightarrow \infty$.

Substituting (7) to the general flow equation (2) for the Hamiltonian (6) we obtain

$$
\begin{aligned}
& \frac{\mathrm{d}}{\mathrm{d} l} \ln \xi_{\boldsymbol{k}}(l)=4\left|\Delta_{\boldsymbol{k}}(l)\right|^{2}, \\
& \frac{\mathrm{d}}{\mathrm{d} l} \ln \Delta_{\boldsymbol{k}}(l)=-4\left(\xi_{\boldsymbol{k}}(l)\right)^{2} .
\end{aligned}
$$

The Equation (9) yields an exponential flow

$$
\Delta_{\boldsymbol{k}}(l)=\Delta_{\boldsymbol{k}} \mathrm{e}^{-4 \int_{0}^{l} \mathrm{~d} l^{\prime}\left(\xi_{\boldsymbol{k}}\left(l^{\prime}\right)\right)^{2}},
$$

and (8) leads to $\xi_{\boldsymbol{k}}(l)=\xi_{\boldsymbol{k}} \mathrm{e}^{4 \int_{0}^{l} \mathrm{~d} l^{\prime}\left|\Delta_{\boldsymbol{k}}\left(l^{\prime}\right)\right|^{2}}$, therefore the off-diagonal term $\Delta_{\boldsymbol{k}}(l)$ vanishes in the limit $l \rightarrow$ $\infty$. Combining (8) and (9) we notice that $\frac{\mathrm{d}}{\mathrm{d} l}\left(\xi_{\boldsymbol{k}}^{2}(l)+\right.$ $\left.\left|\Delta_{\boldsymbol{k}}(l)\right|^{2}\right)=0$ which implies the invariance $\xi_{\boldsymbol{k}}^{2}(l)+$ $\left|\Delta_{k}(l)\right|^{2}=$ const. Due to $\Delta_{k}(\infty)=0$ we conclude that the quasi-particle energies take the following BCS form

$$
\tilde{\xi}_{\boldsymbol{k}}=\operatorname{sgn}\left(\xi_{\boldsymbol{k}}\right) \sqrt{\xi_{\boldsymbol{k}}^{2}+\left|\Delta_{\boldsymbol{k}}\right|^{2}},
$$

where the asymptotic value is denoted by $\tilde{\xi}_{\boldsymbol{k}} \equiv$ $\lim _{l \rightarrow \infty} \xi_{\boldsymbol{k}}(l)$.

\section{Linear response theory}

We now apply the same procedure for studying the electrodynamic properties of the superconductor. In presence of a weak electromagnetic field $\boldsymbol{A}(\boldsymbol{r}, t)$ the linear response is given by an integral relation between the vector potential $\boldsymbol{A}$ and the induced current $\boldsymbol{J}(\boldsymbol{r}, t)$

$$
\begin{aligned}
& \boldsymbol{J}(\boldsymbol{r}, t)=-\int \mathrm{d} \boldsymbol{r}^{\prime} \int_{-\infty}^{t} \mathrm{~d} t^{\prime} K\left(\boldsymbol{r}-\boldsymbol{r}^{\prime}, t-t^{\prime}\right) \\
& \quad \times \boldsymbol{A}\left(\boldsymbol{r}^{\prime}, t^{\prime}\right) .
\end{aligned}
$$

Specific properties of the medium are contained in the kernel $K\left(\boldsymbol{r}-\boldsymbol{r}^{\prime}, t-t^{\prime}\right)$. The Fourier transform of this kernel function consists of the diamagnetic and paramagnetic contributions [5]

$$
K_{\alpha, \beta}(\boldsymbol{q}, \omega)=\frac{n e^{2}}{m} \delta_{\alpha, \beta}+e^{2} \Pi_{\alpha, \beta}(\boldsymbol{q}, \omega),
$$

where $\alpha, \beta$ are $x, y, z$ coordinates.

Introducing the imaginary time $\tau$ we can express the paramagnetic term by the following current-current Green's function

$$
\Pi_{\alpha, \beta}(\boldsymbol{q}, \tau) \equiv-\left\langle\hat{T}_{\tau} \hat{\boldsymbol{j}}_{\boldsymbol{q}, \alpha}(\tau) \hat{\boldsymbol{j}}_{-\boldsymbol{q}, \beta}\right\rangle,
$$

where the current operator $\hat{j}_{\boldsymbol{q}}$ is defined as

$$
\hat{\boldsymbol{j}}_{\boldsymbol{q}}=\sum_{\boldsymbol{k}, \boldsymbol{q}} \boldsymbol{v}_{\boldsymbol{k}+\frac{\boldsymbol{q}}{2}} \hat{c}_{\boldsymbol{k}, \sigma}^{\dagger} \hat{c}_{\boldsymbol{k}+\boldsymbol{q}, \sigma},
$$

and velocity $\boldsymbol{v}_{\boldsymbol{k}}=\nabla_{\boldsymbol{k}} \varepsilon_{\boldsymbol{k}}$.

In order to re-derive the kernel of BCS superconductor [5] we start the analysis of the initial derivative

$$
\left(\frac{\mathrm{d} \hat{\boldsymbol{j}}_{\boldsymbol{q}}^{\sigma}(l)}{\mathrm{d} l}\right)_{l=0}=\left[\hat{\eta}(l), \hat{\boldsymbol{j}}_{\boldsymbol{q}}^{\sigma}(l)\right]_{l=0},
$$

for each spin configuration. Using the generating operator $(7)$ we find that

$$
\begin{aligned}
& \left(\frac{\mathrm{d} \hat{\boldsymbol{j}}_{\boldsymbol{q}}^{\uparrow}(l)}{\mathrm{d} l}\right)_{l=0}=2 \sum_{\boldsymbol{k}} \boldsymbol{v}_{\boldsymbol{k}+\frac{\boldsymbol{q}}{2}} \\
& \times\left(\xi_{\boldsymbol{k}} \Delta_{\boldsymbol{k}} \hat{c}_{-\boldsymbol{k}, \downarrow} \hat{c}_{\boldsymbol{k}+\boldsymbol{q}, \uparrow}+\xi_{\boldsymbol{k}+\boldsymbol{q}} \Delta_{\boldsymbol{k}+\boldsymbol{q}} \hat{c}_{\boldsymbol{k}, \uparrow}^{\dagger} \hat{c}_{-\boldsymbol{k}-\boldsymbol{q}, \downarrow}^{\dagger}\right), \\
& \left(\frac{\mathrm{d} \hat{\boldsymbol{j}}_{\boldsymbol{q}}^{\downarrow}(l)}{\mathrm{d} l}\right)_{l=0}=-2 \sum_{\boldsymbol{k}} \boldsymbol{v}_{\boldsymbol{k}+\frac{\boldsymbol{q}}{2}} \\
& \times\left(\xi_{\boldsymbol{k}} \Delta_{\boldsymbol{k}} \hat{c}_{-\boldsymbol{k}, \uparrow} \hat{c}_{\boldsymbol{k}+\boldsymbol{q}, \downarrow}+\xi_{\boldsymbol{k}+\boldsymbol{q}} \Delta_{\boldsymbol{k}+\boldsymbol{q}} \hat{c}_{\boldsymbol{k}, \downarrow}^{\dagger} \hat{c}_{-\boldsymbol{k}-\boldsymbol{q}, \uparrow}^{\dagger}\right) .
\end{aligned}
$$

From the Eqs. (17), (18) we infer the following $l$ dependent parametrization of the current operators (taking into account the initial structure and the new terms appearing in the derivatives)

$$
\begin{aligned}
& \hat{\boldsymbol{j}}_{\boldsymbol{q}}^{\uparrow}(l)=\sum_{\boldsymbol{k}} \boldsymbol{v}_{\boldsymbol{k}+\frac{q}{2}}
\end{aligned}
$$

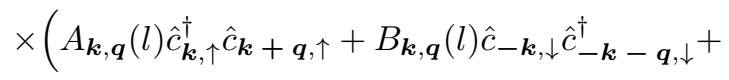

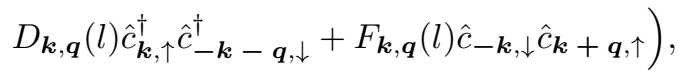

$$
\begin{aligned}
& \hat{j}_{\boldsymbol{q}}^{\downarrow}(l)=\sum_{\boldsymbol{k}} \boldsymbol{v}_{\boldsymbol{k}+\frac{\boldsymbol{q}}{2}} \\
& \times\left(A_{\boldsymbol{k}, \boldsymbol{q}}(l) \hat{c}_{\boldsymbol{k}, \downarrow}^{\dagger} \hat{c}_{\boldsymbol{k}+\boldsymbol{q}, \downarrow}+B_{\boldsymbol{k}, \boldsymbol{q}}(l) \hat{c}_{-\boldsymbol{k}, \uparrow} \hat{c}_{-\boldsymbol{k}}^{\dagger}-\boldsymbol{q}, \uparrow\right. \\
& \left.-D_{\boldsymbol{k}, \boldsymbol{q}}(l) \hat{c}_{\boldsymbol{k}, \downarrow}^{\dagger} \hat{c}_{-\boldsymbol{k}}^{\dagger}-\boldsymbol{q}, \uparrow-F_{\boldsymbol{k}, \boldsymbol{q}}(l) \hat{c}_{-\boldsymbol{k}, \uparrow} \hat{c}_{\boldsymbol{k}}+\boldsymbol{q}, \downarrow\right),
\end{aligned}
$$

with the initial values $A_{\boldsymbol{k}, \boldsymbol{q}}(0)=1$ and $B_{\boldsymbol{k}, \boldsymbol{q}}(0)=$ $D_{\boldsymbol{k}, \boldsymbol{q}}(0)=F_{\boldsymbol{k}, \boldsymbol{q}}(0)=0$. In the next step, we insert the ansatz (19), (20) in the flow equation for the current operator

$$
\frac{\mathrm{d} \hat{\boldsymbol{j}}_{\boldsymbol{q}}^{\sigma}(l)}{\mathrm{d} l}=\left[\hat{\eta}(l), \hat{\boldsymbol{j}}_{\boldsymbol{q}}^{\sigma}(l)\right] .
$$

By inspecting the terms on left and right hand side of the flow equation (21) we derive the following set of flow equations for all $l$-dependent coefficients

$$
\begin{aligned}
& \frac{\mathrm{d} A_{\boldsymbol{k}, \boldsymbol{q}}(l)}{\mathrm{d} l}=-2\left(\xi_{\boldsymbol{k}+\boldsymbol{q}} \Delta_{\boldsymbol{k}+\boldsymbol{q}} D_{\boldsymbol{k}, \boldsymbol{q}}(l)\right. \\
& \left.+\xi_{\boldsymbol{k}} \Delta_{\boldsymbol{k}} F_{\boldsymbol{k}, \boldsymbol{q}}(l)\right) \\
& \frac{\mathrm{d} B_{\boldsymbol{k}, \boldsymbol{q}}(l)}{\mathrm{d} l}=2\left(\xi_{\boldsymbol{k}} \Delta_{\boldsymbol{k}} D_{\boldsymbol{k}, \boldsymbol{q}}(l)\right. \\
& \left.\quad+\xi_{\boldsymbol{k}+\boldsymbol{q}} \Delta_{\boldsymbol{k}+\boldsymbol{q}} F_{\boldsymbol{k}, \boldsymbol{q}}(l)\right) \\
& \frac{\mathrm{d} D_{\boldsymbol{k}, \boldsymbol{q}}(l)}{\mathrm{d} l}=2\left(\xi_{\boldsymbol{k}+\boldsymbol{q}} \Delta_{\boldsymbol{k}+\boldsymbol{q}} A_{\boldsymbol{k}, \boldsymbol{q}}(l)\right. \\
& \left.\quad-\xi_{\boldsymbol{k}} \Delta_{\boldsymbol{k}} B_{\boldsymbol{k}, \boldsymbol{q}}(l)\right) \\
& \frac{\mathrm{d} F_{\boldsymbol{k}, \boldsymbol{q}}(l)}{\mathrm{d} l}=2\left(\xi_{\boldsymbol{k}} \Delta_{\boldsymbol{k}} A_{\boldsymbol{k}, \boldsymbol{q}}(l)\right. \\
& \left.-\xi_{\boldsymbol{k}+\boldsymbol{q}} \Delta_{\boldsymbol{k}+\boldsymbol{q}} B_{\boldsymbol{k}, \boldsymbol{q}}(l)\right) .
\end{aligned}
$$

From these Eqs. (22)-(25) we find that 


$$
\begin{aligned}
& \frac{\mathrm{d}}{\mathrm{d} l}\left(A_{\boldsymbol{k}, \boldsymbol{q}}(l)+B_{\boldsymbol{k}, \boldsymbol{q}}(l)\right)=2\left(\xi_{\boldsymbol{k}+\boldsymbol{q}} \Delta_{\boldsymbol{k}+\boldsymbol{q}}-\xi_{\boldsymbol{k}} \Delta_{\boldsymbol{k}}\right) \\
& \quad \times\left(F_{\boldsymbol{k}, \boldsymbol{q}}(l)-D_{\boldsymbol{k}, \boldsymbol{q}}(l)\right), \\
& \frac{\mathrm{d}}{\mathrm{d} l}\left(F_{\boldsymbol{k}, \boldsymbol{q}}(l)-D_{\boldsymbol{k}, \boldsymbol{q}}(l)\right)=-2\left(\xi_{\boldsymbol{k}+\boldsymbol{q}} \Delta_{\boldsymbol{k}+\boldsymbol{q}}-\xi_{\boldsymbol{k}} \Delta_{\boldsymbol{k}}\right) \\
& \quad \times\left(A_{\boldsymbol{k}, \boldsymbol{q}}(l)+B_{\boldsymbol{k}, \boldsymbol{q}}(l)\right) .
\end{aligned}
$$

For clarity we shall now introduce the compact notation

$$
\begin{aligned}
& A_{\boldsymbol{k}, \boldsymbol{q}}(l)+B_{\boldsymbol{k}, \boldsymbol{q}}(l) \equiv x_{\boldsymbol{k}, \boldsymbol{q}}(l), \\
& F_{\boldsymbol{k}, \boldsymbol{q}}(l)-D_{\boldsymbol{k}, \boldsymbol{q}}(l) \equiv y_{\boldsymbol{k}, \boldsymbol{q}}(l) .
\end{aligned}
$$

From Eqs. (26), (27) we obtain the following invariance

$$
\left|x_{\boldsymbol{k}, \boldsymbol{q}}(l)\right|^{2}+\left|y_{\boldsymbol{k}, \boldsymbol{q}}(l)\right|^{2}=\text { const. }=1 .
$$

This invariance is useful when determining the asymptotic $l \rightarrow \infty$ values of both parameters $x_{\boldsymbol{k}, \boldsymbol{q}}(l)$ and $y_{\boldsymbol{k}, \boldsymbol{q}}(l)$. We can rewrite (26) as

$$
\frac{\mathrm{d} x_{\boldsymbol{k}, \boldsymbol{q}}(l)}{y_{\boldsymbol{k}, \boldsymbol{q}}(l)}=2\left(\xi_{\boldsymbol{k}+\boldsymbol{q}} \Delta_{\boldsymbol{k}+\boldsymbol{q}}-\xi_{\boldsymbol{k}} \Delta_{\boldsymbol{k}}\right) \mathrm{d} l,
$$

and we further integrate both sides (31) in the limits $\int_{l=0}^{l=\infty}$. Using $y_{\boldsymbol{k}, \boldsymbol{q}}(l)=\sqrt{1-\left(x_{\boldsymbol{k}, \boldsymbol{q}}(l)\right)^{2}}$ we get for the 1.h.s.

$$
\int_{l=0}^{l=\infty} \frac{\mathrm{d} x_{\boldsymbol{k}, \boldsymbol{q}}(l)}{\sqrt{1-\left(x_{\boldsymbol{k}, \boldsymbol{q}}(l)\right)^{2}}}=-\arccos \left(x_{\boldsymbol{k}, \boldsymbol{q}}(\infty)\right) .
$$

Integrating the r.h.s. of the Eq. (31), where $l$-dependent $\xi_{\boldsymbol{k}}$ and $\Delta_{\boldsymbol{k}}$ obey the following invariance $\left|\xi_{\boldsymbol{k}}(l)\right|^{2}+$ $\left|\Delta_{\boldsymbol{k}}(l)\right|^{2}=$ const. $=\left|\xi_{\boldsymbol{k}}(\infty)\right|^{2}$, we obtain

$$
\begin{gathered}
\int_{l=0}^{l=\infty} 2\left(\xi_{\boldsymbol{k}+\boldsymbol{q}} \Delta_{\boldsymbol{k}+\boldsymbol{q}}-\xi_{\boldsymbol{k}} \Delta_{\boldsymbol{k}}\right) \mathrm{d} l \\
\quad=-\frac{1}{2}\left(\arccos \left(\frac{\left|\Delta_{\boldsymbol{k}}\right|}{\left|\xi_{\boldsymbol{k}}(\infty)\right|}\right)\right. \\
\left.\quad-\arccos \left(\frac{\left|\Delta_{\boldsymbol{k}+\boldsymbol{q}}\right|}{\left|\xi_{\boldsymbol{k}+\boldsymbol{q}}(\infty)\right|}\right)\right) .
\end{gathered}
$$

From these integrals (32) and (33) we get

$$
\begin{aligned}
& 2 \arccos \left(x_{\boldsymbol{k}, \boldsymbol{q}}(\infty)\right)=\arccos \left(\frac{\Delta_{\boldsymbol{k}+\boldsymbol{q}} \Delta_{\boldsymbol{k}}}{\xi_{\boldsymbol{k}+\boldsymbol{q}}(\infty) \xi_{\boldsymbol{k}}(\infty)}\right. \\
& \left.+\sqrt{1-\frac{\Delta_{\boldsymbol{k}+\boldsymbol{q}}^{2}}{\xi_{\boldsymbol{k}+\boldsymbol{q}}^{2}(\infty)}} \sqrt{1-\frac{\Delta_{\boldsymbol{k}}^{2}}{\xi_{\boldsymbol{k}}^{2}(\infty)}}\right),
\end{aligned}
$$

and finally determine the $l=\infty$ parameters

$$
\begin{aligned}
& {\left[\tilde{x}_{\boldsymbol{k}, \boldsymbol{q}}\right]^{2}=\frac{1}{2}\left(1+\frac{\Delta_{\boldsymbol{k}+\boldsymbol{q}} \Delta_{\boldsymbol{k}}+\xi_{\boldsymbol{k}+\boldsymbol{q}} \xi_{\boldsymbol{k}}}{\tilde{\xi}_{\boldsymbol{k}+\boldsymbol{q}} \tilde{\xi}_{\boldsymbol{k}}}\right),} \\
& {\left[\tilde{y}_{\boldsymbol{k}, \boldsymbol{q}}\right]^{2}=\frac{1}{2}\left(1-\frac{\Delta_{\boldsymbol{k}+\boldsymbol{q}} \Delta_{\boldsymbol{k}}+\xi_{\boldsymbol{k}+\boldsymbol{q}} \xi_{\boldsymbol{k}}}{\tilde{\xi}_{\boldsymbol{k}+\boldsymbol{q}} \tilde{\xi}_{\boldsymbol{k}}}\right),}
\end{aligned}
$$

where we used the abbreviation $\tilde{\alpha} \equiv \lim _{l \rightarrow \infty} \alpha(l)$. These solutions (35), (36) correspond to the usual BCS coherence factors $\left(\tilde{u}_{\boldsymbol{k}+\boldsymbol{q}} \tilde{u}_{\boldsymbol{k}}+\tilde{v}_{\boldsymbol{k}+\boldsymbol{q}} \tilde{v}_{\boldsymbol{k}}\right)^{2}$ and $\left(\tilde{u}_{\boldsymbol{k}+\boldsymbol{q}} \tilde{v}_{\boldsymbol{k}}-\tilde{v}_{\boldsymbol{k}+\boldsymbol{q}} \tilde{u}_{\boldsymbol{k}}\right)^{2}$.

Due to the diagonal form of the transformed Hamiltonian $\hat{H}(\infty)$ one can easily calculate the current-current response function (14) at arbitrary temperature $T$. The final expression takes the following form

$$
\begin{aligned}
& \Pi_{\alpha, \beta}(\boldsymbol{q}, \mathrm{i} \nu)=\sum_{\boldsymbol{k}} \boldsymbol{v}_{\boldsymbol{k}+\frac{\boldsymbol{q}}{2}, \alpha} \boldsymbol{v}_{\boldsymbol{k}+\frac{\boldsymbol{q}}{2}, \beta} \\
& \quad \times\left(\left[\tilde{x}_{\boldsymbol{k}, \boldsymbol{q}}\right]^{2}\left(f_{F D}\left(\tilde{\xi}_{\boldsymbol{k}+\boldsymbol{q}}\right)-f_{F D}\left(\tilde{\xi}_{\boldsymbol{k}}\right)\right)\right. \\
& \quad \times\left(\frac{1}{\mathrm{i} \nu+\tilde{\xi}_{\boldsymbol{k}+\boldsymbol{q}}-\tilde{\xi}_{\boldsymbol{k}}}-\frac{1}{\mathrm{i} \nu-\tilde{\xi}_{\boldsymbol{k}+\boldsymbol{q}}+\tilde{\xi}_{\boldsymbol{k}}}\right) \\
& \quad+\left[\tilde{y}_{\boldsymbol{k}, \boldsymbol{q}}\right]^{2}\left(1-f_{F D}\left(\tilde{\xi}_{\boldsymbol{k}+\boldsymbol{q}}\right)-f_{F D}\left(\tilde{\xi}_{\boldsymbol{k}}\right)\right) \\
& \left.\quad \times\left(\frac{1}{\mathrm{i} \nu-\tilde{\xi}_{\boldsymbol{k}+\boldsymbol{q}}-\tilde{\xi}_{\boldsymbol{k}}}-\frac{1}{\mathrm{i} \nu+\tilde{\xi}_{\boldsymbol{k}+\boldsymbol{q}}+\tilde{\xi}_{\boldsymbol{k}}}\right)\right),
\end{aligned}
$$

where $f_{F D}(\omega)=\left(\exp \left(\omega / k_{B} T\right)+1\right)^{-1}$ is the Fermi-Dirac distribution function. Equation (37) rigorously reproduces the BCS response function [5]. In the static $\omega=0$ and long wave-length limit $\boldsymbol{q} \rightarrow 0$ the response function has a nonvanishing value (for $T<T_{c}$ ) and in consequence the relation (13) implies the Londons' equation describing the Meissner effect [5] $\hat{\boldsymbol{j}}_{\boldsymbol{q}}=-\boldsymbol{A}(\boldsymbol{q}) \frac{n_{s}(T) e^{2}}{m c}$, where $n_{s}(T)$ is the superfluid density.

\section{Further outlook}

The flow equation technique is useful for investigating the linear response of the BCS superconductor but one can generalize it also to determine the current-current response function of the electron systems with strong pairing fluctuations. We have applied such approach to the boson-fermion model and examined the pseudogap state where the preformed pairs are incoherent above $T_{c}$ [4]. We were motivated by the experimental data of the torque magnetometry [6] revealing the residual diamagnetism in cuprates above $T_{c}$. We have analyzed the response function in the static limit and found a clear evidence for the pronounced diamagnetic contribution, which might be relevant to the experimental data obtained for the underdoped cuprate materials [6-8].

Nonperturbative scheme of the flow equation method can be also used to deal with nonequilibrium phenomena of the correlated systems. One can for instance calculate the transient and steady currents beyond the linear response regime for the interacting quantum impurities (described by the Anderson-type models). In the long time limit the steady state is reached uniformly using the expansion with respect to the interaction term [9]. The real time evolution problems of the quantum many body systems might be determined from the corresponding flow equations.

The nonequilibrium spin dynamics has been also studied for the spin-boson model [10]. The stable, long-time behavior has been obtained from the forward-backward unitary transformation, giving the nonperturbative solution of the Heisenberg equations of motion for an operator [10]. The equilibrium and nonequilibrium Kondo model has been investigated so far in the weak-coupling 
regime. The flow equation approach allowed to estimate the static and dynamical quantities, including their leading logarithmic corrections [11].

We thus conclude that the flow equation technique allows for studying the symmetry broken state within the linear response framework and offers a possibility to go beyond such limitation. This nonperturbative aspect is very important whenever the many-body effects are strong and one has to consider the quantum fluctuations beyond the usual gaussian corrections.

\section{Acknowledgments}

This work is partly supported by the Polish Ministry of Science and Education under the grant NN202 263138.

\section{References}

[1] F. Wegner, Ann. Phys. (Leipzig) 3, 77 (1994).

[2] S.D. Głazek, K.G. Wilson, Phys. Rev. D 49, 4214 (1994); S.D. Głazek, K.G. Wilson, Phys. Rev. D 48, 5863 (1993).
[3] S. Kehrein, The flow equation approach to manyparticle systems, Springer Tracts Mod. Phys., Vol. 215, Berlin 2006.

[4] M. Zapalska, T. Domański, Phys. Rev. B 84, 174520 (2011).

[5] A.L. Fetter, J.D. Walecka, Quantum theory of manyparticle systems, McGraw-Hill Book Company, New York 1971; J.R. Schrieffer, Theory of superconductivity, W.A. Benjamin Inc. Publisher, New York 1964.

[6] L. Li, Y. Wang, S. Komiya, S. Ono, Y. Ando, G.D. Gu, N.P. Ong, Phys. Rev. B 81, 054510 (2010).

[7] T. Iye, T. Nagatochi, R. Ikeda, A. Matsuda, J. Phys. Soc. Jpn. 79, 114711 (2010).

[8] E. Bernardi, A. Lascilfari, A. Ragimonti, L. Romano, M. Scavini, C. Oliva, Phys. Rev. B 81, 064502 (2010).

[9] P. Wang, S. Kehrein, Phys. Rev. B 82, 125124 (2010).

[10] A. Hackl, S. Kehrein, Phys. Rev. B 78, 092303 (2008).

[11] P. Fritsch, S. Kehrein, Phys. Rev. B 81, 035113 (2010). 